\title{
Preheating of Fermions
}

\author{
Patrick B. Greene \\ Department of Physics and Astronomy, University of Hawaii, 2505 Correa Rd., Honolulu HI 96822, USA \\ Lev Kofman \\ Institute for Astronomy, University of Hawaii, 2680 Woodlawn Dr., Honolulu, HI 96822, USA
}

(February 1, 2008)

\begin{abstract}
In inflationary cosmology, the particles constituting the Universe are created after inflation in the process of reheating due to their interaction with the oscillating inflaton field. In the bosonic sector, the leading channel of particle production is the non-perturbative regime of parametric resonance, preheating, during which bosons are created exponentially fast. Pauli blocking prohibits the unbounded creation of fermions. For this reason, it has been silently assumed that the creation of fermions can be treated with perturbation theory for the decay of individual inflatons. We consider the production of fermions interacting with the coherently oscillating inflatons. We find that the actual particle production occurs in a regime of the parametric excitation of fermions, leading to preheating of fermions. Fermion preheating differs significantly from the perturbative expectation. It turns out that the number density of fermions varies periodically with time. The total number of fermions quickly saturates to an average value within a broad range of momenta $\propto q^{1 / 4}$, where $q$ is the usual resonance parameter. The resonant excitation of fermions may affect the transfer inflaton energy, estimations of the reheating temperature, and the abundance of superheavy fermions and gravitinos. Back in the bosonic sector, outside of the parametric resonance bands there is an additional effect of parametric excitation of bosons with bounded occupation numberin the momentum range $\propto q^{1 / 4}$.
\end{abstract}

PACS: $98.80 . \mathrm{Cq} \quad$ UH-IfA-98-44

In the inflationary scenario, the Universe initially expands quasi-exponentially in a vacuum-like state with vanishing temperature, entropy, and particle number densities. Consider a simple chaotic inflation during which all energy is contained in the inflaton field $\phi$ which is slowly rolling down to the minimum of its effective potential $V(\phi)$. When inflation ends at $\phi \sim M_{p}$, the inflaton field begins to oscillate near the minimum of $V(\phi)$ with a very large amplitude, $\phi \simeq \frac{1}{10} M_{p}$. This scalar field can be considered as a coherent superposition of $\phi$-quasiparticles with zero momenta, i.e. inflatons at rest.

In this scenario, all the particles constituting the Universe are created due to their interactions with the inflatons. For a toy model describing the interaction between inflatons and other Bose particles $\chi$, we may consider the term $g^{2} \phi^{2} \chi^{2}$ or $g^{2} \sigma \phi \chi^{2}$ in the Lagrangian. For Fermi particles $\psi$ we can include a Yukawa coupling $h \bar{\psi} \phi \psi$ to the inflaton. If we assume for simplicity that the bare masses of the fields $\chi$ and $\psi$ are very small, the inflatons will decay into ultra-relativistic particles. If the creation of these particles is sufficiently slow, the particles will simultaneously interact with each other and come to a state of thermal equilibrium at the reheating temperature $T_{r}$. The theory of this gradual reheating, developed long ago with the first models of inflation [1], was based on the perturbative theory with respect to the coupling constants $g^{2}$ and $h$. Consider a quantum Bose field $\chi$ with the eigenfunctions $\chi_{k}(t) e^{+i \mathbf{k} \cdot \mathbf{x}}$ for comoving momenta $\mathbf{k}$. The temporal part of the eigenfunction obeys an oscillator-like equation with a time-dependent frequency: $\Omega_{k}^{2}(t)=\frac{k^{2}}{a^{2}}+g^{2} \phi(t)^{2}$ or $g^{2} \sigma \phi(t)$. The iterative solution to the equation of motion for $\chi_{k}(t)$ gives the perturbation theory result for the particles' occupation number: $n_{k} \propto g^{4} \ll 1$. The resulting rate for the three-legs process $\phi \rightarrow \chi \chi$ is given by $\Gamma_{\phi \rightarrow \chi \chi}=\frac{g^{4} \sigma^{2}}{8 \pi m_{\phi}}$. By the same methods, one finds for the process $\phi \rightarrow \psi \psi$ a rate $\Gamma_{\phi \rightarrow \psi \psi}=\frac{h^{2} m_{\phi}}{8 \pi}$. Each of the created quanta carries away half of an inflaton's energy, $\frac{m_{\phi}}{2}$. This perturbative result can be interpreted as individual inflatons decaying independently of each other into pairs of $\chi$ or $\psi$-particles.

However, it was recognized that reheating can begin with a stage of boson production in a regime of parametric resonance [2]. Indeed, in the equation for the eigenmode $\chi_{k}(t)$, inflatons act not as individual particles but as a coherently oscillating field $\phi(t)$. The smallness of the coupling $g^{2}$ alone does not necessarily correspond to small occupation number $n_{k}$. The oscillating effective frequency $\Omega_{k}(t)$ results in the parametric amplification of the modes: $\chi_{k} \sim e^{\mu_{k} t}$. In this case, the energy of the inflatons is explosively transferred to the bose field. This process of bosonic preheating occurs very rapidly and far from thermal equilibrium.

For fermions, the Pauli exclusion principle prohibits the occupation number from exceeding $1 / 2$. For this reason, it has been assumed that fermions are created in the perturbative regime with the production rate $\Gamma_{\phi \rightarrow \psi \psi}$ and occupation number $n_{k} \propto h^{2} \ll 1$, as described in the 
classical papers [1].

Let us, however, consider the Dirac equation for a massless quantum Fermi field $\psi(t, \vec{x})$ :

$$
\left[i \gamma^{\mu} \nabla_{\mu}-h \phi(t)\right] \psi=0 .
$$

Here, the inflatons producing fermions also act not as individual particles but as a coherently oscillating field $\phi(t)$. Therefore, we should check whether coherently acting inflatons produce the same number of fermions as an ensemble of individually decaying inflatons. It turns out that the actual production of fermions from the oscillating inflaton field dramatically differs from the perturbative approximation.

We begin our investigation with the model $\frac{1}{4} \lambda \phi^{4}+$ $h \bar{\psi} \phi \psi$. A special feature of this theory is that the problem of fermion production by the inflaton $\phi$ in an expanding universe can be completely reduced to a similar problem in Minkowski space-time. Indeed, let us perform a conformal transformation of the involved fields, $\varphi \equiv a \phi$ and $\Psi \equiv a^{3 / 2} \psi$, and use a conformal time variable, $\tau \equiv \sqrt{\lambda \tilde{\varphi}^{2}} \int \frac{d t}{a(t)}$. Then (without decay) the amplitude of the transformed field $\varphi$ is constant, $\tilde{\varphi}$. The background inflaton solution is given by an elliptic function $\varphi(\tau) \approx \tilde{\varphi} c n\left(\tau, \frac{1}{\sqrt{2}}\right)$ and the period of the background oscillations $T \approx 7.416$ in this model [4].

The equation for the eigenfunctions of the quantum fluctuations in this theory can be reduced to a secondorder equation for an auxillary field $X(\tau, \vec{x})$, so that $\Psi=\left[i \gamma^{\mu} \nabla_{\mu}+h \varphi\right] X$. The eigenmodes of the auxillary field have the form $X_{k}(\tau) e^{+i \mathbf{k} \cdot \mathbf{x}} R_{r}$, where the $R_{r}$ are eigenvectors of the Dirac matrix $\gamma^{0}$ with eigenvalue +1 . The temporal part of the eigenmode obeys an oscillatorlike equation with a complex frequency which depends periodically on time

$$
\ddot{X}_{k}+\left(\kappa^{2}+q f^{2}-i \sqrt{q} \dot{f}\right) X_{k}=0 .
$$

The comoving momentum $k$ enters the equation in the combination $\frac{k^{2}}{\lambda \tilde{\varphi}^{2}} \equiv \kappa^{2}$; therefore, the natural units of momentum are $\sqrt{\lambda} \tilde{\varphi}$. The background oscillations enter in the form $f(\tau)=c n\left(\tau, \frac{1}{\sqrt{2}}\right)$ having unit amplitude. The combination of the coupling parameters $\frac{h^{2}}{\lambda} \equiv q$ ultimately defines the solutions to Eq. (2).

Equation (2) will be the master equation in our investigation. We choose the vacuum positive-frequency initial condition $X_{k}\left(\tau_{0}\right)=\mathcal{N}_{k} e^{-i \kappa \tau}$. The normalization factor is $\mathcal{N}_{k}=\left(2 \Omega_{k}\left(\Omega_{k}+\sqrt{q} f\right)\right)^{-1 / 2}$ with the real part of the effective frequency given by $\Omega_{k}^{2} \equiv \kappa^{2}+q f(\tau)^{2}$. Notice that the problem of the production of bose particles $\chi$ in the theory $\frac{1}{4} \lambda \phi^{4}+\frac{1}{2} g^{2} \phi^{2} \chi^{2}$ is reduced to an equation similar to (2) but without the imaginary part of the frequency and with the resonance parameter $q=\frac{g^{2}}{\lambda}$ 糔.

Using the standard $\Psi$-field operator expansion, we can express the comoving occupation number of particles in a given spin state through the solution of Eq. (2) (see, e.g., [5] for details)

$$
n_{k}(\tau)=\frac{1}{2}-\frac{\kappa^{2}}{\Omega_{k}} \operatorname{Im}\left(X_{k} \dot{X}_{k}^{*}\right)-\frac{\sqrt{q} f}{2 \Omega_{k}} .
$$

Certainly $n_{k} \leq 1 / 2$. The total comoving number density of particles and antiparticles in both spin states is $\frac{4}{(2 \pi)^{3}} \int d^{3} k n_{k}$. Hence, the energy density of created fermions is $\epsilon_{\psi}=\frac{1}{2 \pi^{3}} \int d^{3} k \Omega_{k} n_{k}$.

Eq. (2) can be solved numerically and the occupation number of particles can be found with (3). The results for $n_{k}(\tau)$ with different parameters $q$ are plotted in Fig. 11.

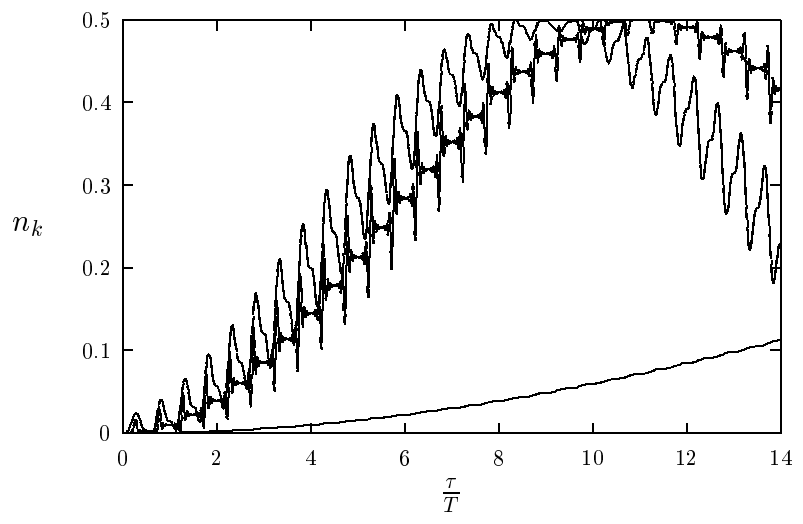

FIG. 1. The occupation number $n_{k}$ in $\lambda \phi^{4}$-inflation as a function of time $\tau$ (in units of $T$ ) for $q \equiv \frac{h^{2}}{\lambda}=10^{-4}$ (lower), 1 (middle on right), and 100 (upper on right) and $\kappa^{2}=0.18,1.11$, and 11.9 , respectively. The period of the modulation $\frac{\pi}{\nu_{k}}$ (see Eq. (身)) is about 88, 20 and 22 (in units of $T$ ) accordingly.

The occupation number exhibits high frequency (period $<\frac{T}{2}$ ) oscillations which are modulated by a long period behavior. If we average the occupation number over these high frequency oscillations, $\bar{n}_{k}(\tau)=$ $\frac{1}{T} \int_{\tau}^{(\tau+T)} d \tau n_{k}(\tau)$, we can write the smoothed occupation number of fermions in a factorized form

$$
\bar{n}_{k}(\tau)=F_{k} \sin ^{2} \nu_{k} \tau .
$$

It is remarkable that, for all $q$, the occupation number of fermions evolves periodically with time [6]. The spectrum (envelope) function $F_{k}$ and the period $\frac{\pi}{\nu_{k}}$ depend on the parameter $q$. Typically $\frac{\pi}{\nu_{k}} \sim 10-100$, see Fig. 司. Therefore, the fermionic modes get fully exited around their time-average value $\frac{F_{k}}{2}$ within just tens of inflaton oscillations! This is very different from the result of the perturbative approximation which gives the timing for excitation $\sim \Gamma_{\phi \rightarrow \psi \psi}^{-1}$ which is about $10^{14}$ inflaton oscillations [3]. Notice that for $q \gg 1$, the number of fermions is almost constant between two successive zeros of the inflaton field. It jumps in a step-like manner at instances when $\phi(t)$ crosses zero. This is a familiar picture for the creation of bosons explained in 3,4.1. However, contrary to the bosonic resonance which is unbounded, the 
fermionic resonance reaches its peak value $F_{k} \leq 1 / 2$ after a time $\frac{\pi}{2 \nu_{k}}$ and then falls back to zero, where it begins the cycle again.

Fortunately, there is a semi-analytic shortcut for the calculation of $F_{k}$ and $\nu_{k}$ [7]. We found that the envelope function is given by the expression

$$
F_{k}=\frac{1}{\sin ^{2} \nu_{k} T} \frac{\kappa^{2}}{2 \Omega_{k}^{2}}\left(\operatorname{Im} X_{k}^{(1)}(T)\right)^{2} .
$$

Here, $X_{k}^{(1)}(T)$ is the value of the first fundamental solution of Eq. (2) (defined by the intial conditions $X_{k}^{(1)}(0)=$ $\left.1, \dot{X}_{k}^{(1)}(0)=0\right)$ taken after one full oscillation; it is a function of $k$ which depends on the parameter $q$. The modulation frequency $\nu_{k}$ is given by the relation $\cos \nu_{k} T=$ $-\operatorname{Re} X_{k}^{(1)}(T)$. Therefore, to find $F_{k}$ and $\nu_{k}$, one need only calculate the complex value $X_{k}^{(1)}(T)$ instead of performing a full numerical integration of Eq. (2).

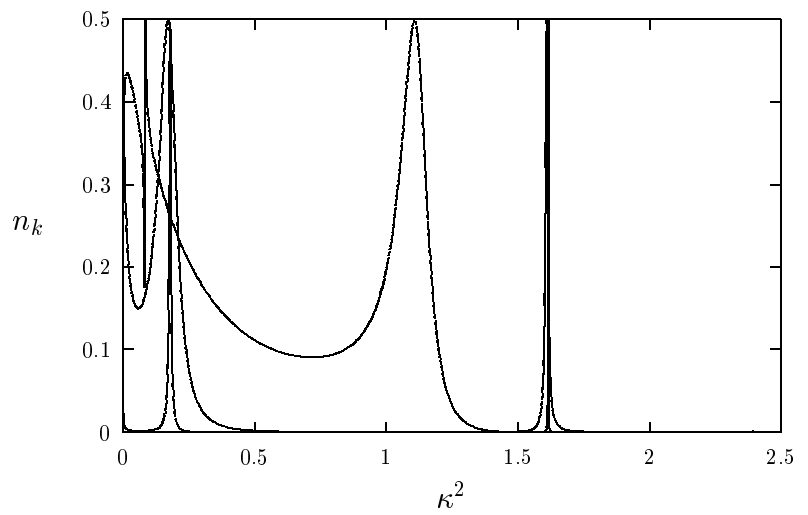

FIG. 2. The envelope functions $F_{k}$ showing the bands of fermion resonance excitation in $\lambda \phi^{4}$-inflation for $q \equiv \frac{h^{2}}{\lambda}=10^{-4}, 10^{-2}$, and 1.0 (the narrowest to broadest band, respectively). The band in the case $q=10^{-2}$ already deviates considerably from the perturbative expectation.

We calculated $X_{k}^{(1)}(T)$ numerically and constructed the envelope function $F_{k}$ plotted in Fig. 2. The spectrum of $n_{k}$ is very different from what is expected from the perturbative calculations, where it is narrowly peaked around $\kappa=\frac{\pi}{T} \approx 0.424$ with the width $\Gamma_{\phi \rightarrow \psi \psi}^{-1}$. In Figs. 3 t 4 we show, using (5), how the fermionic resonance bands are filled after 10 background oscillations.

The function $\nu_{k}$ gives us the time scale for fermion excitation. In Fig. 同 we plot the period of modulation $\frac{\pi}{\nu_{k}}$ as a function of $k$. This function is peaked where $F_{k}$ is peaked, i.e. the peaks of the resonance curve are the last to fill. For $q \gtrsim 1$, the period for modes in the principle peak is about $20 T$. When $q \ll 1$, modes in the principle peak grow more slowly, with a period of approximately $\frac{T}{\sqrt{q}}$. Interestingly, the period of modes out of the $F_{k}$-peaks is significantly shorter. For this reason, the integrated number of fermions is quickly saturated to its time-average value.

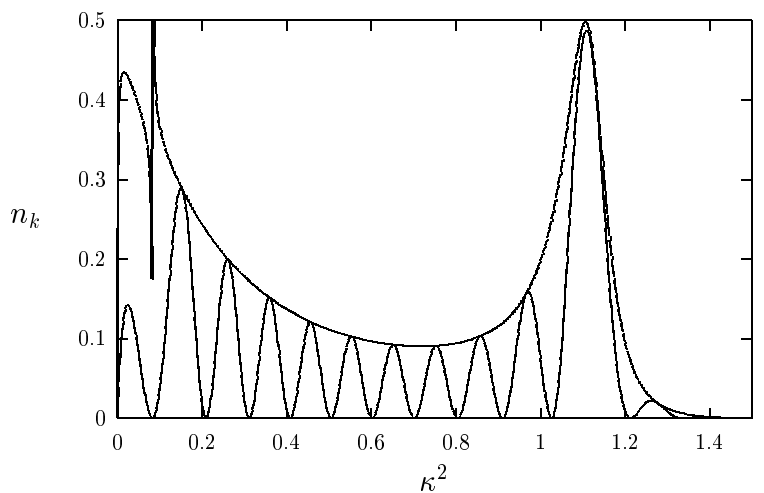

FIG. 3. The resonance excitation band of fermions in $\lambda \phi^{4}$-inflation for $q \equiv \frac{h^{2}}{\lambda}=1.0$. The heavy line shows the envelope $F_{k}$ of maximum occupation number calculated from Eq. (E). The light curve is the actual occupation number $n_{k}$ of each mode, calculated from Eq. (4), after 10 background oscillations.

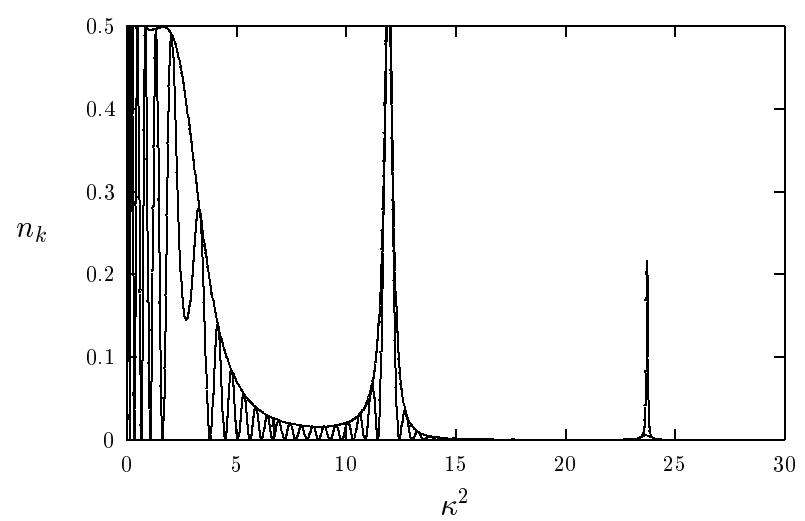

FIG. 4. As in Fig. 3, the resonance excitation band in $\lambda \phi^{4}$-inflation for $q \equiv \frac{h^{2}}{\lambda}=100.0$. The heavy line shows the envelope $F_{k}$. The light curve is the actual occupation number of each mode $n_{k}$ after 10 background oscillations.

The huge differences in the spectrum and rate of excitation of fermions produced by the coherently oscillating background field vs. those in the naive perturbative calculations are due to the interference of the fermion fluctuations. In this respect we can talk about the parametric excitation of fermions, where $q$ plays the role of the resonance parameter, and the frequency $\nu_{k}$ can be viewed as the fermionic conterpart of the bosonic characteristic exponent $\mu_{k}$. The amplitude of $n_{k}$ shows a distinct sequence of resonance bands whose widths sharply narrow with increasing zone number. This is again reminiscent

\footnotetext{
${ }^{*}$ Notice that, for the resonant bands in the bosonic case, the modulating factor in Eq. (何) is replaced by $\sinh ^{2} \mu_{k} t$. This leads to the familiar estimation $n_{k} \sim e^{2 \mu_{k} t}$ for the number of bosons.
} 
of the usual bosonic resonance. For $q \ll 1$, the resonance bands are located at $\kappa=\frac{\pi}{T}(2 l+1)$, where $l=0,1,2, \ldots$.

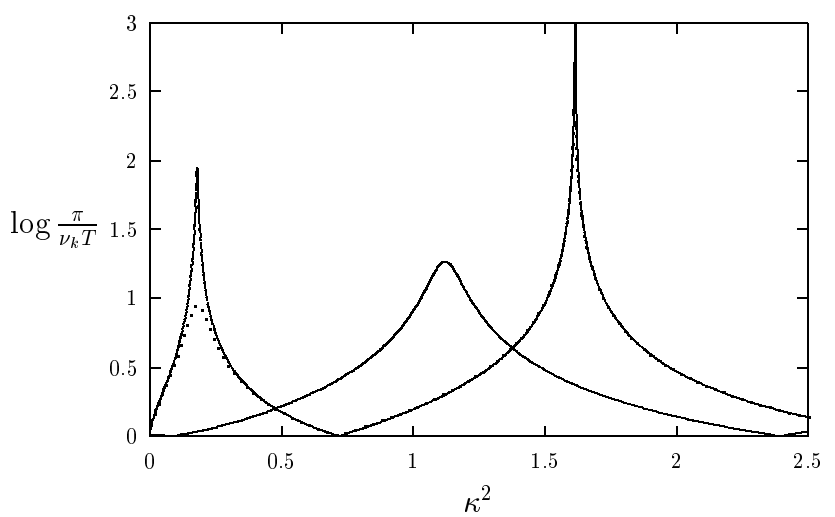

FIG. 5. The $\log$ of the period of modulation $\frac{\pi}{\nu_{k}}$ (in units of $T$ ) as a function of $\kappa^{2}$ for $q \equiv \frac{h^{2}}{\lambda}=10^{-4}, 10^{-2}$, and 1.0 for the light, dotted, and heavy curves respectively.

An important issue is the width of the parametric excitation of fermions. From Fig. 2 we notice that for any $q$ the first, broadest zone has the width $\Delta \kappa \sim q^{1 / 4}$. This can be understood from the condition for nonadiabatic excitation of particles, $\dot{\Omega}_{k} \geq \Omega_{k}^{2}$, which gives us $\Delta k \leq q^{1 / 4} \sqrt{\lambda} \tilde{\varphi}$. This is similar to how the result was elaborated for the broad bosonic resonance [3]. There are also very narrow zones with higher momenta, which are also quickly saturated. This may be important for the production of superheavy fermions with a mass $m_{\psi} \sim q^{1 / 4} m_{\phi}$, where the effective inflaton mass $m_{\phi} \simeq 10^{13} \mathrm{Gev}$.

Let us estimate the energy that will be accumulated in created fermions. Averaging over short-period oscillations, we have

$$
\bar{\epsilon}_{\psi}=\frac{2}{\pi^{2}} \int d k k^{2} F_{k} \sin ^{2} \nu_{k} \tau .
$$

For the most interesting case of the broad resonance excitation, $q \gg 1$, we get $\epsilon_{\psi} \sim 0.1 h^{2} q^{1 / 4} \epsilon_{\phi}$, where the inflaton energy is $\epsilon_{\phi}=\frac{1}{4} \lambda \tilde{\varphi}^{4}$. In chaotic $\frac{1}{4} \lambda \phi^{4}$-inflation, $\lambda \simeq 10^{-13}$. If the resonance parameter $q$ is large but the coupling parameter is small, $h \leq 0.1$, then only a small fraction of the inflaton energy will be transferred into fermions. However, if we push the parameters to $h \gtrsim 0.3\left(q \gtrsim 10^{12}\right)$, the inflaton energy can be transferred into fermions. This is quite an extreme case, because it requires SUSY cancellation of the radiative corrections $\sim h^{4} \phi^{4}$. However, in the hybrid inflation scenario, for example, there is more freedom to chose the model parameters which control the efficiency of preheating [8]. There may be a range of parameters for which the inflaton energy is fully converted into fermions even before the bosonic resonance develops.

Let us now turn to the model $\frac{1}{2} m^{2} \phi^{2}+h \bar{\psi} \phi \psi$, where $m$ is the inflaton mass. In this inflationary model the inflaton evolution is simply described by harmonic oscillations, $\phi \approx \Phi(t) \sin m t$, with decreasing amplitude, $\Phi(t)=\frac{M_{p}}{\sqrt{3 \pi} m t}$. However, the problem of fermion production in this model cannot be reduced to a problem in Minkowski space-time; the expansion of the universe is essential. Let us assume, for a moment, that there is no expansion and the amplitude $\Phi(t)=$ const. Then the master equation would be Eq. (2) where now the resonance parameter $q \equiv \frac{h^{2} \Phi^{2}}{m^{2}}$ and $\kappa^{2} \equiv \frac{k^{2}}{m^{2}}$ (c.f. the bosonic resonance [3]). The result for the occupation number of fermions in this case can also be found with Eq. (5) and is plotted in Fig. 6.

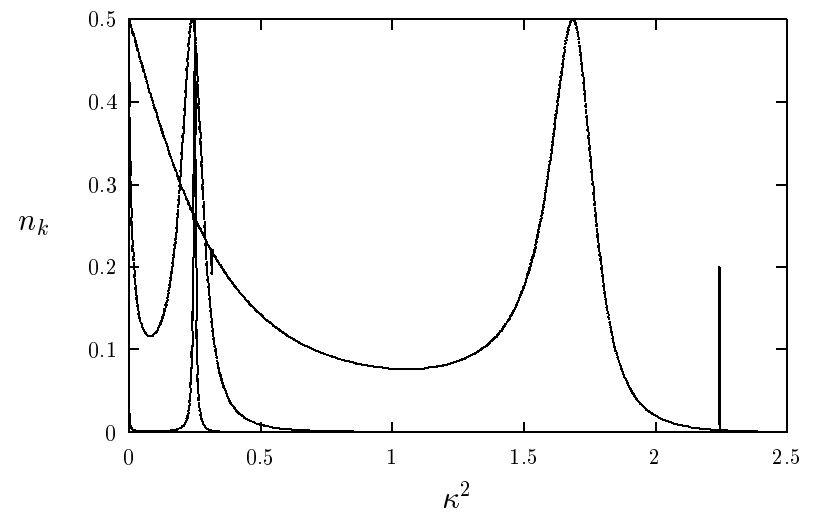

FIG. 6. The resonance band in $m^{2} \phi^{2}$-inflation with $q \equiv \frac{h^{2} \Phi^{2}}{m^{2}}=10^{-4}, 10^{-2}$, and 1.0 for the narrowest to broadest band, respectively. The band in the case $q=10^{-2}$ already deviates considerably from the perturbative expectation.

Without expansion of the universe, the qualitative result is similar to that for the conformal theory. However, if we take the expansion into account, we can expect an adiabatic change in the structure of the fermion parametric excitation. This is similar to the erasure of differences between resonant and non-resonant modes and the onset of stochastic resonance in the bosonic case [3]. Therefore, we conjecture that, for the massive inflaton, the parametric excitation of fermions fills a Fermi-sphere of radius $\Delta k \sim q^{1 / 4} m$ without distinct resonance zones.

If expansion redshifts the fermion modes fast enough, it can prevent them from being resonantly excited. We found that for $q_{0} \leq 1$ or $h \leq 10^{-6}$ we return to the perturbation theory result for the rate of fermion pair production $\frac{1}{a^{4}} \frac{d}{d t}\left(a^{4} n_{\bar{\psi} \psi}\right) \approx \Gamma_{\phi \rightarrow \psi \psi} n_{\phi}$, where the comoving number density of inflatons is $n_{\phi}=m \Phi^{2} a^{3} / 2$. This is to be interpreted as the creation of a pair of fermions, $\bar{\psi}$ and $\psi$, from the decay of a single inflaton at the instant of resonance between a given momentum $k$ and inflaton mass $m, k=\frac{1}{2} m a(t)$.

In this paper we studied the production of fermions by an oscillating inflaton field, and found that fermions are created in a regime of parametric excitation, which is very different from the perturbative regime. This can be important to many interesting cosmological applica- 
tions including the channel of inflaton energy transfer, the estimation of the reheating temperature, the production of superheavy fermions, the production of gravitinos in the supergravity inflationary models [9], etc. It also elucidates another feature of bosonic preheating, missed in the previous studies. Indeed, in the resonance bands bosons are exponentially unstable, $n_{k} \sim e^{2 \mu_{k} t}$. However, outside of the resonance bands $\mu_{k}$ is imaginary and we will have bounded excitation of bosonic fluctuations $n_{k} \sim O(1)$ given by Eq. (4). How this will alter the preheating scenario and its lattice simulation, based on classical approximation of fluctuations [10], shall be seen.

The authors are grateful to Andrei Linde for fruitful discussions, and organizers of the CAPP98 Workshop at CERN, June 7-12, where the results of the paper were reported This work was supported by NSF grant AST9529-225. After this work was completed, we learned about related report 11].

[1] A.D. Dolgov and A.D. Linde, Phys. Lett. 116B, 329 (1982); L.F. Abbott, E. Fahri and M. Wise, Phys. Lett. 117B, 29 (1982); A.A. Starobinsky, in: Proc. Quantum Theory of Gravity, eds. M.A. Markov \& P.C. West (Plenum, N.Y., 1984), p. 103.

[2] L. A. Kofman, A. D. Linde, and A. A. Starobinsky, Phys. Rev. Lett. 73, 3195 (1994).

[3] L. A. Kofman, A. D. Linde, and A. A. Starobinsky, Phys. Rev. D56, 3258 (1997).

[4] P. B. Greene, L. Kofman, A. D. Linde, and A. A. Starobinsky, Phys. Rev. D 56, 6175 (1997); D. Boyanovsky, H.J. de Vega, R. Holman, \& J.F.J. Salgado, Phys. Rev. D547570 (1996).

[5] A.D. Dolgov and D.P. Kirilova, Sov. J. Nucl. Phys., 51, 172 (1990).

[6] For an arbitrary periodic background field $f(\tau)$, it can be shown that (4) is an exact formula for the (non-averaged) values $n_{k}(N T)$ but only after precisely $N$ background oscillations, $\tau=N T$. This matches the numerical curves of Fig. 1 at these instants. This result, which is also relevant for preheating, was first derived for an external electromagnetic field in: N.B. Narozhnyi and A.I. Nikishov, Sov.Phys. JETP, 38, 427 (1974); V.S. Popov, Sov. J. Nucl. Phys., 19, 1140 (1974).

[7] To derive (f) for preheating, we have extended significantly the method developed for pair production from the vacuum by an external electric field in: M.V. Mostepanenko and V.M. Frolov, Sov. J. Nucl. Phys., 19, 885 (1974).

\footnotetext{
${ }^{\dagger}$ See http://wwwth.cern.ch/capp98/programme.htm for transparencies.
}

[8] J. García-Bellido \& A. Linde, Phys. Rev.D57 6065 (1998).

[9] J. Ellis \& D.N. Nanopoulos, Phys. Lett. 116B, 133 (1982); K. Olive, Phys. Rep. 190, 308 (1990).

[10] S. Khlebnikov \& I. Tkachev, Phys. Rev. Lett. 77219 (1996).

[11] J. Baacka, K. Heitmann \& C. Pätzold, hep-ph/9806205 